\title{
EFFECTS OF IXERIS CHINENSIS (THUNB.) NAKAI BOILING WATER EXTRACT ON HEPATITIS B VIRAL ACTIVITY AND HEPATOCELLULAR CARCINOMA
}

\section{Ko-Nien Shih ${ }^{1 *}$, Wen-Tao Huang ${ }^{1}$, Chung-Liang Chang $^{2}$, Chih-Chung Feng ${ }^{3}$}

${ }^{1}$ Department of Radiological Technology, College of Medical Sciences and Technology, Yuanpei University, HsinChu City 30015, Taiwan. ${ }^{2}$ Department of Environmental Engineering and Health, College of Health Sciences, Yuanpei University, HsinChu City 30015, Taiwan, ${ }^{3}$ Cardiovascular Internal Medicine Department, Changhua Christian Hospital, Changhua, 50006, Taiwan.

*E-mail: shihkn@mail.ypu.edu.tw

\begin{abstract}
Background: Hepatitis B virus (HBV) infection and hepatocellular carcinoma are major diseases that affect the Taiwanese population. Therefore, the development of an alternative herbal medicine that can effectively treat these diseases is a research target. In this study, we tested Ixeris Chinensis (Thunb.) Nakai boiling water extract (ICTN BWE) in vitro and analysed its effects on the HBV and liver cancer.

Materials and Methods: We used a human liver cancer cell line (Hep3B, a cell line that continuously secretes HBV particles into a medium) as an experimental model for the screening of various ICTN BWE concentrations and their effects on the HBV in vitro.

Results: Our results showed that $75 \mu \mathrm{g} / \mathrm{mL}$ ICTN BWE downregulated the relative expression of the hepatitis B virus surface antigens (HBsAg) to $77.1 \%$. Using the human liver cancer cell lines HuH-7 and HepG2, and 3-(4,5- dimethylthiazol-zyl)-2,5-diphenyl tetrazolium bromide (MTT) and tumour clonogenic assays, we then showed that ICTN BWE inhibits hepatocellular carcinoma growth.

Conclusion: Fluorescent microscopy of DAPI(4',6-Diamidino-2-phenylindole)-stained nuclei and DNA fragmentation assays confirmed the inhibitory effects of ICTN BWE on liver tumour cell growth through induction of apoptosis.
\end{abstract}

Keywords: herbal medicine, Ixeris Chinensis (Thunb.) Nakai, antihepatocellular carcinoma, apoptosis, antihepatitis B virus

\section{Introduction}

Hepatitis B infection and hepatocellular carcinoma represent severe public health problems worldwide, and the HBV is the leading cause of both diseases (Perz et al., 2006; Beasley, 2009). Several anti-viral drugs have been certified for the treatment of hepatitis B. However, they cause significant complications such as dose-dependent side-effects and drug resistance. Therefore, novel safe and effective anti-HBV agents are urgently required to treat patients with chronic HBV infection. Several studies have shown that Chinese herbs have the potential to exert antivirus and anticancer effects (Liao et al., 2010; Chen et al., 2011; Wang et al., 2012; Cao et al., 2013). In this study, we investigated a herb, ICTN, whichdisplays anti-HBV and anti-hepatocellular carcinoma activities.

ICTN is a perennial herb commonly found in Taiwan. It is extensively distributed in the plains and mountains of moderate elevation, and grows on roadsides, ridges, or on uncultivated land. The entire plant contains a milky juice. Taxonomically, it belongs to the Compositae plant family and the Ixeris genus. It exists in Taiwan, the United States, South Korea, Japan, and China. The traditional medical application of ICTN is treatment of pneumonia, bronchitis, and diarrhoea. ICTN displays antipyretic, analgesic, and anti-inflammatory activities, and is used in folk medicine for the treatment of liver disease (Kan, 1981).

Previous studies have shown that ICTN exerts hepatoprotective effects and can treat liver disease (Qiusheng et al., 2004; Lin et al., 1994). In addition to effectively suppressing the hyperplasia of inflammatory human mesangial cells (Kuo et al., 1998), it displays anticancer and antiviral properties. In previous studies, ICTN suppressed the growth of prostate cancer (PC-3; Khalil et al., 2005) and lung cancer (VA-13; Zhang et al., 2006) cells by mediating apoptosis. It also inhibited the metastatic activity of prostate cancer cells (Khalil et al., 2005), and 
http://dx.doi.org/10.4314/ajtcam.v11i1.30

effectively suppressed leukaemia cell growth (K562) and HSV-1 and HSV-2 activity (Chiang et al., 2004).

Until now the effects of ICTN on the hepatitis virus and on hepatocellular carcinoma have not been well described. In this study, we observed that ICTN BWE inhibited the secretion of hepatitis B surface antigens and suppressed the growth of hepatocellular carcinoma lines. In addition, the morphology of DAPI-stained cells and DNA fragmentation assay using gel electrophoresis showed clear fragmentation in the chromatin and DNA condensation within the nucleus of cells treated with ICTN BWE, indicating that ICTN BWE suppresses liver cancer cell growth by inducing apoptosis.

\section{Materials and Methods Preparation of ICTN BWE}

An entire ICTN plant was dried in an oven at $45{ }^{\circ} \mathrm{C}$ for $24 \mathrm{~h}$. A high-speed grinding machine was then used to grind the plant before passing it through a 40-mesh sieve. Ten grams of filtered granular ICTN were placed into $300 \mathrm{~mL}$ of $100{ }^{\circ} \mathrm{C}$ boiling water and heated for 30 min. Centrifugation was performed after cooling (rotational speed $6000 \mathrm{rpm}$, temperature $25^{\circ} \mathrm{C}$ ) to separate the extract from the plant residues. Whatman filter paper was then used for filtering, and the filtered crude extract was stored at $-84^{\circ} \mathrm{C}$ overnight. The frozen crude extract was placed in a freeze-dryer, which converted it to a dry powder. Paraffin paper was used to seal and protect the powder from light in a $-20^{\circ} \mathrm{C}$ refrigerator. This boiling water extract was used in subsequent experiments.

\section{Cell lines and cell culture conditions}

Hep3B, which is a human hepatocellular carcinoma cell line and squamous cell carcinoma, contains a chromosome in which the entire hepatitis B virus genome is integrated. It can secrete hepatitis B virus particles, and represents a cell platform for study of the hepatitis B virus. $\mathrm{HuH7}$ is a human hepatocellular carcinoma cell line and squamous cell carcinoma. This cell line was derived from a tumour from the liver of a 57-year-old Japanese man in 1982. HepG2 is a human hepatocellular carcinoma cell line and hepatoblastoma. It is a human liver cancer cell line with a high degree of differentiation, retaining the cell type of a normal human liver cell. All cell lines were cultivated in a Dulbecco's Modified Eagle's Medium (DMEM; Biowest). A 10\% inactivated fetal bovine serum (GIBCO), $100 \mathrm{U} / \mathrm{mL}$ penicillin, $100 \mu \mathrm{g} / \mathrm{mL}$ streptomycin, $2 \mathrm{mM}$ L-glutamine, and $1 \%$ non-essential amino acid were added to each cell culture and cultivated in a $37{ }^{\circ} \mathrm{C} 5 \%$ carbon dioxide cell incubator. When subculturing the cells, a phosphate buffered saline (PBS) was used for washing (twice), and then trypsin-EDTA (Ethylene diamine tetraacetic acid) was applied for 3 to $5 \mathrm{~min}$ (the duration differed according to the cell line). This caused the shedding of cells from the petri dish after which they were broken up using pipettes. Finally, the cells were separated into new petri dishes, and a DMEM culture fluid was added.

\section{Hepatitis B virus surface antigens enzyme-linked immunosorbent assay (HBsAg ELISA)}

The kit for testing HBsAg (SURASE B-96; TMB) was purchased from the General Biological Corporation of Taiwan, and all experimental steps were performed according to the kit's instruction manual. After incubating ICTN BWE $(0,20,50$, or $100 \mu \mathrm{g} / \mathrm{mL})$ with Hep3B cells for $72 \mathrm{~h}$, the top layer of the culture fluid was extracted for the testing of HBsAg. A 450/650 nm ELISA Plate Reader was used to measure absorbance, and to determine the amount of HBsAg secretion. The number of cells in each group of cells was calculated using haemocytometry. The data obtained from absorbance and the number of cells indicated the relative expression of HBsAg, with the data of the control group assigned as $100 \%$ to provide a standard for comparison.

\section{(4) 3-(4,5-Dimethylthiazol-zyl)- 2, 5-diphenylterazolium bromide (MTT) assay}

Cells were seeded at a density of $3 \times 10^{5}$ cells/well in a 6 -well cell culture plate, and cultivated for $48 \mathrm{~h}$ with ICTN BWE of various concentrations (0 to $0.5 \mathrm{mg} / \mathrm{mL}$ ). Approximately $0.5 \mathrm{mg} / \mathrm{mL}$ MTT was then added to the petri dish and incubated for $4 \mathrm{~h}$. DMSO (Dimethyl sulfoxide) was used to crystallise and dissolve formazan (purple). A spectrophotometer was used at a wavelength of $570 \mathrm{~nm}$ to determine the tumour cell survival rate. 


\section{Tumour clonogenic assay}

Trypsin was used to process the HuH-7 cells into a single cell suspension, which was seeded into a 6-well culture plate at 1000 cells/well. Triplicate control and drug-treated plates were prepared and cultured for $24 \mathrm{~h}$ at $37{ }^{\circ} \mathrm{C}$, allowing the cells to attach to the plate. Various concentrations of ICTN BWE ( 0 to $0.5 \mathrm{mg} / \mathrm{mL}$ ) were used to treat the cells for $48 \mathrm{~h}$, after which cells were washed 3 times using a PBS. The cells were cultured at $37{ }^{\circ} \mathrm{C}$ for a further $9 \mathrm{~d}$ to allow cell colony formation. After colony formation, the culture liquid was removed and washed 3 times with PBS. A fixative (methanol: acetic acid = 3:1) was used to fix the cells, and a $0.5 \%$ crystal violet methanol solution was used for staining. After removal of the dye by washing in water, more than 50 cell colonies were counted under a microscope.

\section{Fluorescence microscopy and DNA electrophoresis}

First, cells were seeded to a cover slip. After treating with $350 \mu \mathrm{g} / \mathrm{mL}$ or $500 \mu \mathrm{g} / \mathrm{mL}$ ICTN BWE for $72 \mathrm{~h}$, the cells were rinsed in a PBS and fixed in $4 \%$ paraformaldehyde for $15 \mathrm{~min}$. Then, DAPI $(1 \mu \mathrm{g} / \mathrm{mL})$ was added at room temperature for $15 \mathrm{~min}$. A PBS was used for washing, after which cells were mounted in a mounting solution. An inverted fluorescence microscope was used to observe and record the changes in nuclear morphologies. After the collection of groups of cells, a genomic DNA purification kit (QIAGEN, Germany) was used to purify the cell DNA. Five micrograms of DNA from each group of cells was extracted, and electrophoresis analysis was performed in a $1.8 \%$ agarose gel. After using EtBr (ethidium bromide) for staining, the DNA fragments were observed and photographed under a UV light box.

\section{Statistical analysis}

Sigma Plot 10.0 statistical software was used for statistical analysis. Data are expressed as mean $\pm \mathrm{SD}$, and a paired $t$-test was used to compare each group with the control group. A $p$-value $<0.05$ was considered significant.

\section{Results}

\section{ICTN BWE downregulates HBsAg expression in Hep3B cells}

Human Hep3B cells carry multiple copies of HBV DNA that are stably integrated into the host cell genome. They distinctly resemble infected human liver cells, therefore offering a suitable cell system for chronic HBV infection. In this study, we used Hep3B cells as a test platform to evaluate the effects of ICTN BWE on the expression of HBsAg and to elucidate its hepatoprotective mechanisms. Before we tested the effect of ICTN BWE on HBsAg expression in vitro, we first investigated whether ICTN BWE has a direct toxic effect on the Hep3B cell line. The cells were incubated with ICTN BWE at graded concentrations of 0 (vehicle control), 10, 20, 50, and $100 \mu \mathrm{g} / \mathrm{mL}$. The cytotoxicity of ICTN BWE on Hep3B cells was evaluated by using MTT assay. Hep3B cells had the same survival rate as the vehicle control in the presence of 10-100 $\mu \mathrm{g} / \mathrm{mL}$ of ICTN BWE (data not shown). Thus, we used the mentioned experimental data as reference concentrations to investigate the effect of ICTN BWE on the production of HBeAg by HepG3B cells. The supernatant was collected after treatment with different concentrations of ICTN BWE at different times, and the titre of HBsAg was determined by using ELISA. Our results indicated a dose-dependent downregulation of relative HBsAg expression (to 76.8\%) in the experimental group treated with $75 \mu \mathrm{g} / \mathrm{mL}$ ICTN BWE compared to the untreated control group (Figure 1). The treated cells exhibited no evidence of cytotoxicity, indicating that, in undamaged liver cells, low concentrations of ICTN BWE can inhibit HBsAg production in vitro.

ICTN BWE inhibits the growth of hepatocellular carcinoma cells

We also investigated whether ICTN BWE has a direct effect on the growth of human liver tumour cells in vitro. HuH-7 and HepG2 human 
http://dx.doi.org/10.4314/ajtcam.v11i1.30

liver tumour cells were treated with ICTN BWE at different concentrations for $48 \mathrm{~h}$, and the cells were harvested for MTT assays to evaluate the viability of the tumour cells. Our results showed that ICTN BWE dose-dependently inhibited the growth of the two liver cancer cell lines. The antiproliferative effect of ICTN BWE was especially notable in HuH-7 cells compared to HepG2 cells. In HuH-7 cells, the inhibition rates were 89.6\% (200 $\mu \mathrm{g} / \mathrm{mL}), 73.9 \%(350 \mu \mathrm{g} / \mathrm{mL})$, and 50.5\% (500 $\mu \mathrm{g} / \mathrm{mL})$. In HepG2 cells, the inhibition rates were $92.2 \%(200 \mu \mathrm{g} / \mathrm{mL}), 79.6 \%(350$ $\mu \mathrm{g} / \mathrm{mL}$ ), and $77.9 \%(500 \mu \mathrm{g} / \mathrm{mL})$ (Figure 2). The difference between the two cell viabilities is as high as $27.4 \%$ at a high concentration $(500$ $\mu \mathrm{g} / \mathrm{mL})$ of ICTN BWE.

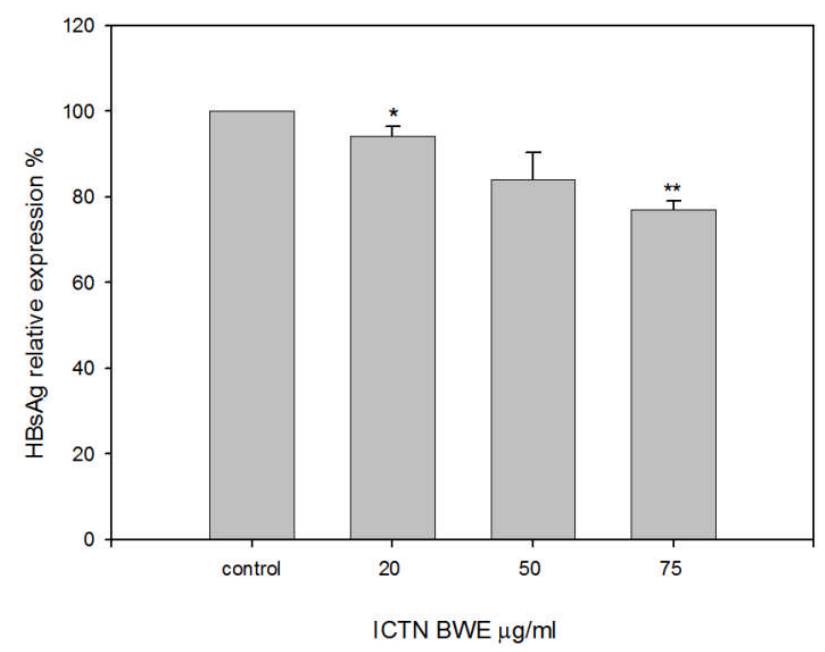

Figure 1: ICTN BWE could dose-dependently inhibit the expression of HBsAg. HBsAg expression in ICTN BWE-treated Hep3B cells was evaluated using the SURASE B-96 (TMB) ELISA kit. Columns, mean $(\mathrm{n}=3)$; bars, SE. Paired $t$-test: *, $p<0.05, * *, p<0.01$

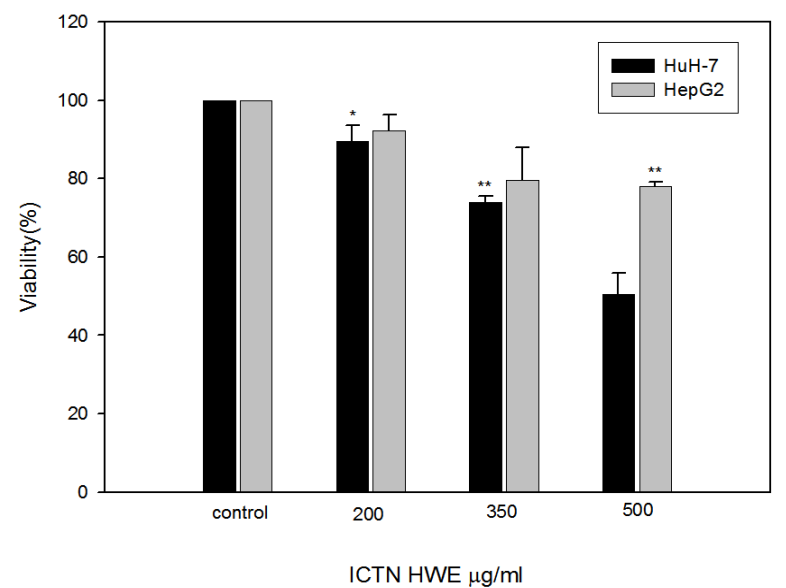

Figure 2: Growth-inhibiting effects of ICTN BWE on human liver tumour cells in vitro. HuH-7 and HepG2 cells were treated with the indicated concentrations of ICTN BWE for $48 \mathrm{~h}$, and then cell viability was evaluated using MTT assay. Columns, mean ( $\mathrm{n}=3$ ); bars, SE. Paired $t$-test: $*, p<0.05, * *, p<0.01$

\section{The cytotoxicity of ICTN BWE to HuH-7 cells was examined with clonogenic assay}

Clonogenic assay is an in vitro cell survival assay based on the ability of a single tumour cell to grow into a colony (at least 50 cells). The assay tests every tumour cell in the population for its ability to undergo unlimited division, which is a phenotype of tumour cells. This method is usually used to determine the cytotoxicity of tumour cells exposed to various cytotoxic agents (Franken et al., 2006). Using a control group assigned as $100 \%$ for comparison, our results showed that $500 \mu \mathrm{g} / \mathrm{mL}$ ICTN BWE reduced the colony formation rate of HuH-7 cells to $52.6 \%$, and the inhibitory effect of ICTN BWE on colony formation was dose-dependent (Figure 3). The results of clonogenic assay indicate that ICTN 
http://dx.doi.org/10.4314/ajtcam.v11i1.30

BWE inhibits the growth of hepatocellular carcinoma cells.

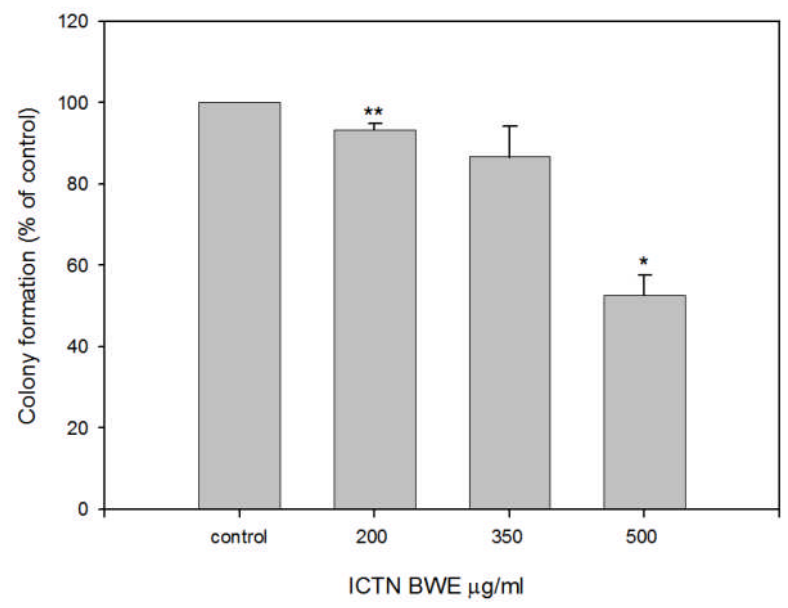

Figure 3: Clonogenic assays were used to investigate survival of ICTN BWE treated liver tumour cells. The inhibitory effects of ICTN BWE were evaluated using clonogenic assay in HuH-7 cells. Cell survival was determined following exposure to the indicated ICTN BWE concentrations for $48 \mathrm{~h}$ and normalised to the control cell values. Columns, mean $(\mathrm{n}=3)$; bars, SE. Paired $t$-test: **, $p<0.05$, **, $p<0.01$

\section{ICTN BWE induces apoptosis in HuH-7 hepatoma cell line in vitro}

During apoptosis, endonucleases cleave chromosomal DNA into fragments with a length of approximately 200 bp and multiples. Figure 4A shows the results of a representative experiment in which HuH-7 cells were treated with ICTN BWE at indicated concentrations for $72 \mathrm{~h}$, and genomic DNA were extracted and analysed by DNA electrophoresis. We observed that the control lane contained only high-molecular-weight DNA, but ICTN BWE treatment led to a dose-dependent increase in DNA fragmentation, providing evidence of apoptosis. Next to assess ICTN BWE-induced apoptosis in HuH-7 cells and to examine the influence of ICTN BWE on nuclear apoptotic events, we stained the chromatin of the same treatment groups with DAPI and counted apoptotic nuclei using fluorescence microscopy. Figures 4C and 4D show micrographs of DAPI-stained cells, which have nuclei with fragmented chromatin (white arrow) that are typical for the apoptotic nuclear type. These findings verify that ICTN BWE induces apoptosis in hepatocellular carcinoma cells.

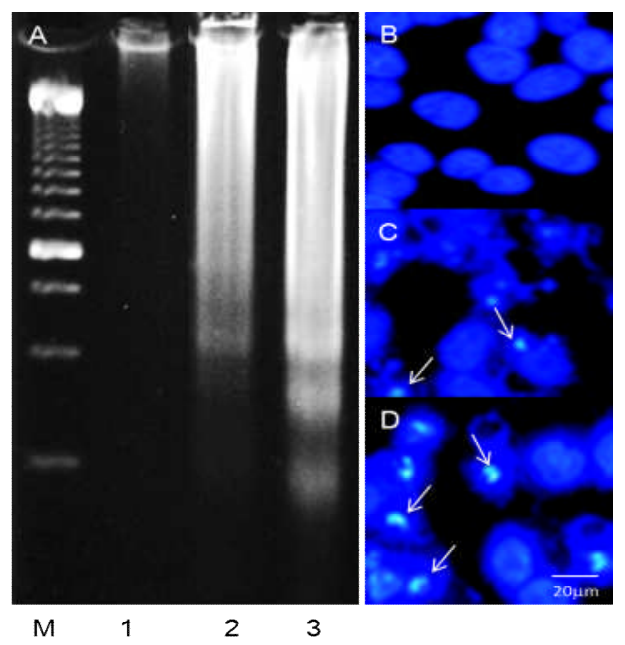

Figure 4: DNA fragmentation and DAPI staining in the ICTN BWE treated HuH-7 hepatoma cell line. Apoptosis was evaluated using (A) DNA fragmentation (Lane 1: control; Lane 2: 350 $\mu \mathrm{g} / \mathrm{mL}$ ICTN BWE; Lane 3: $500 \mu \mathrm{g} / \mathrm{mL}$ ICTN BWE; M: DNA markers) and (B-D) DAPI fluorescent staining (B: control; C: $350 \mu \mathrm{g} / \mathrm{mL}$ ICTN BWE; D: $500 \mu \mathrm{g} / \mathrm{mL}$ ICTN BWE). Arrows indicate condensed nuclear DNA. 
http://dx.doi.org/10.4314/ajtcam.v11i1.30

\section{Discussion}

Although the use of herbal medicine provokes debate concerning its current and future role in healthcare and evidence for both efficacy and safety, numerous herbal medicines have been known to exert hepatoprotective (Zhou et al., 2009; Park et al., 2011) and anticancer (Babykutty et al., 2009; Rand et al., 2009) effects. ICTN is a primary hepatoprotective Chinese medicine with efficacy for treating leukaemia, lung cancer, breast cancer, and nasopharyngeal carcinoma. It also effectively treats cirrhosis of the liver, sore throats, pneumonia, cystitis, urethritis, constipation, and skin disease. Therefore, research of the mechanism by which ICTN protects the liver against hepatocellular carcinoma is critical.

In this study, an ELISA was used to demonstrate that ICTN BWE can reduce the relative expression of $\mathrm{HBsAg}$ (to $76.8 \%$ in $75 \mu \mathrm{g} / \mathrm{mL}$ ) in low-toxicity concentrations in liver cells and likely inhibits HBV replication. The HBV life cycle is a complex process that requires fine tuning of numerous viral and cellular proteins. During replication, the viral HBx protein can increase the cytoplasmic calcium concentration by interacting with the mitochondrial permeability transition pore (MPTP), causing its opening and subsequent outflow of intra-mitochondrial calcium into the cytoplasm. The Src kinase and downstream signal transduction messengers are subsequently activated, thus promoting HBV DNA replication (Rahmani et al., 2000; Bouchard et al., 2001). Xie et al. indicated that cyclosporine A can impair the interaction of the HBx protein with the mitochondria, inhibit subsequent MPTP openings, block the intracellular calcium signalling pathway, and thus inhibit HBV replication (Xie et al., 2007). A previous report revealed that fifteen compounds can be isolated from Ixeris chinensis by using silica gel column chromatography, HPLC, and recrystallization (Wang et al., 2011). We assume that some of these compounds can interact with HBx protein, and thus interfere with the release of mitochondrial calcium into the cytoplasm to cause Src activation, thus inhibiting HBV DNA replication. Our recent unpublished results have shown that ICTN BWE can reduce the cytoplasmic calcium levels of Hep3B cells in a dose-dependent manner, may provide some clues for examining the mechanisms of the inhibitory action of ICTN BWE on HBV replication, and can possibly enable identifying some key factors involved in HBV replication. However, further experiments are required to understand the implications of ICTN BWE in inhibiting HBV replication. Results from MTT assay and tumour colony formation analysis showed that ICTN BWE exerts growth-suppressive effects on the human liver tumour cell lines HuH-7 and HepG2. It more potently inhibited the growth of HuH-7 than HepG2. At an ICTN concentration of 500 $\mu \mathrm{g} / \mathrm{mL}$, the growth-inhibitory effects in HuH-7 and HepG2 cells differed by $27.4 \%$, indicating that ICTN BWE differentially inhibits the growth of different types of hepatocellular carcinoma cells. Our results indicate the potential use of ICTN BWE for treatment of liver tumours similar to the HuH-7 cell type. Using DAPI staining and DNA electrophoresis analysis we showed nuclear morphology and confirmed that ICTN BWE induces apoptosis in liver tumour cells (HuH-7). The mechanism by which ICTN BWE caused the anti-tumour effect through the induction of apoptosis is attractive. Apoptosis plays an important role in eliminating unwanted and potentially dangerous cells, such as tumour cells and cells infected by viruses (Favaloro et al., 2012). At least two broad pathways lead to apoptosis: an extrinsic pathway and an intrinsic pathway. In both pathways, signalling results in the activation of a family of caspases that act in a proteolytic cascade to trigger apoptotic events. Luteolin, a key member of the flavonoid family, is present in ICTN. It exhibits an effect on the inhibition of proliferation and induction of apoptosis in human myeloid leukaemia HL-60 (Ko et al., 2002) and hepatoma HepG2 cells (Lee et al., 2005). Lee et al. (2005) showed that the mitochondrial translocation of Bax/Bak, the activation of JNK, and the release of cytochrome c were critical events in the luteolin-induced apoptosis of human hepatoma HepG2 cells. However, more experiments are required to determine which pathway is involved in ICTN-induced apoptosis in HuH-7 cells. Based on a thorough review of relevant studies, this is the first study to show that ICTN BWE has potential application in the treatment of liver tumours and inhibition of hepatitis B virus activity. More information is required regarding the future clinical applications of ICTN BWE, including preparation, standardisation, identification of active ingredients, and toxicological evaluation.

\section{Acknowledgment}

This study was supported by the Yuanpei University cooperation research plan (100-COMP6011-06).

\section{References}

1. Babykutty, S., Padikkala, J., Sathiadevan, P. P., Vijayakurup, V., Azis, T. K., Srinivas, P., and Gopala, S. (2009). Apoptosis induction of Centella asiatica on human breast cancer cells. Afr J Tradit Complement Altern Med. 6: 9-16. 
http://dx.doi.org/10.4314/ajtcam.v11i1.30

2. Beasley, R. P. Rocks along the road to the control of HBV and HCC. (2009). Ann Epidemiol. 19(4):231-234.

3. Bouchard, M. J., Wang, L. H., Schneider, R. J. (2001). Calcium signaling by HBx protein in hepatitis B virus DNA replication. Science. 294: 2376-2378.

4. Cao, Z., Lin, W., Huang, Z., Chen, X., Zhao, J., Zheng, L., Ye, H., Liu, Z., Liao, L., and Du, J. (2013). Ethyl acetate extraction from a Chinese herbal formula, Jiedu Xiaozheng Yin, inhibits the proliferation of hepatocellular carcinoma cells via induction of G0/G1 phase arrest in vivo and in vitro. Int $\mathbf{J}$ Oncol. 42(1):202-210. Epub 2012 Nov 16.

5. Chen, W., Lim, C. E., Kang, H. J., and Liu, J. (2011). Chinese herbal medicines for the treatment of type A H1N1 influenza: a systematic review of randomized controlled trials. PLoS One. 6(12):e28093.

6. Chiang, L. C., Cheng H. Y., Chen, C. C., Lin, C. C. (2004). In vitro anti-leukemic and antiviral activities of traditionally used medicinal plants in Taiwan. Am J Chin Med. 32: 695-704.

7. Favaloro, B., Allocati, N., Graziano, V., Di Ilio, C., and De Laurenzi, V. (2012). Role of apoptosis in disease. Aging (Albany NY). 4(5):330-349.

8. Franken, N. A., Rodermond, H. M., Stap, J., Haveman, J., and van Bree, C. (2006). Clonogenic assay of cells in vitro. Nat Protoc. 1(5):2315-2319.

9. Kan, W. S. (1981). "Pharmaceutical Botany," National Research Institute of Chinese Medicine, Taipei, Taiwan, p. 562.

10. Khalil, A. T., Shen, Y. C., Guh, J. H., and Cheng, S. Y. (2005). Two new sesquiterpene lactones from Ixeris chinensis. Chem. Pharm. Bull. 53: $15-17$.

11. Ko, W. G., Kang, T. H., Lee, S. J., Kim, Y. C., and Lee, B. H. (2002). Effects of luteolin on the inhibition of proliferation induction of apoptosis in human myeloid leukaemia cells. Phytother. Res. 16: 295-298.

12. Kuo, Y. C., Sun, C. M., Tsai, W. J., Ou, J. C., Chen, W. P., and Lin, C. Y. (1998). Chinese herbs as modulators of human mesangial cell proliferation: preliminary studies. J. Lab. Clin. Med. 132: 76-85.

13. Lee, H. J., Wang, C. J., Kuo, H. C., Chou, F. P., Jean, L. F., and Tseng, T. H. (2005) Induction apoptosis of luteolin in human hepatoma HepG2 cells involving mitochondria translocation of Bax/Bak and activation of JNK. Toxicol Appl Pharmacol. 203(2):124-131.

14. Li, Z., Li, L. J., Sun, Y., and Li, J. (2007). Identification of natural compounds with anti-hepatitis B Virus activity from Rheum palmatum L. ethanol extract. Chemotherapy. 53: 320-326.

15. Liao, H. F., Lu, M. C., Chang, H. C., Wei, C. C., Kao, C. H., Chen, Z. H., Huang, C. C., and Li, C. (2010). Effects of herbal medicinal formulas on suppressing viral replication and modulating immune responses. Am J Chin Med. 38(1):173-190.

16. Lin, S. C., Lin, C. C., Lin, Y. H., and Yao, C. J. (1994). Hepatoprotective effects of Taiwan folk medicine: Ixeris chinensis (Thunb.) Nak. on experimental liver injuries. Am J Chin Med. 22: 243-254.

17. Park J, Kim HY, Lee SM. (2011). Protective effects of Moutan Cortex Radicis against acute hepatotoxicity. Afr J Tradit Complement Altern Med. 8(5 Suppl):220-225.

18. Perz, J. F., Armstrong, G. L., Farrington, L. A., Hutin, Y. J., and Bell, B. P. (2006). The contributions of hepatitis B virus and hepatitis C virus infections to cirrhosis and primary liver cancer worldwide. J Hepatol. 45(4):529-538.

19. Qiusheng, Z., Xiling, S., Xubo, Meng, S., and Changhai, W., (2004). Protective effects of luteolin-7-glucoside against liver injury caused by carbon tetrachloride in rats. Pharmazie. 59: 286-289.

20. Rahmani, Z., Huh, K.W., Lasher, R., and Siddiqui, A., (2000). Hepatitis B virus X protein colocalizes to mitochondria with a human voltage-dependent anion channel, HVDAC3, and alters its transmembrane potential. J Virol. 74: 2840-2846.

21. Rand, R. H., Faridah, A., Ahmed, S. A., Fatemeh, J, Fatimah, A. B., and Zamberi, S. (2009). A review: cancer research of natural products in Asia. Int. J. Cancer Res. 5: 69-82.

22. Wang, J. L., Lv, D., Liang, X. Y., Zhao, M., and Zhang S. J. (2011). Study on chemical constituents of the Ixeris chinensis. Zhong Yao Cai. 34(11):1706-1708.

23. Wang, S., Wu, X., Tan, M., Gong, J., Tan, W., Bian, B., Chen, M., and Wang, Y. (2012). Fighting fire with fire: poisonous Chinese herbal medicine for cancer therapy. J Ethnopharmacol. 140(1):33-45.

24. Xie, H. Y., Xia, W. L., Zhang, C. C., Wu, L. M., Ji, H. F., Cheng, Y., and Zheng, S. S. (2007). Evaluation of hepatitis B virus replication and proteomic analysis of HepG2.2.15 cell line after cyclosporine A treatment. Acta Pharmacol Sin. 28(7):975-984.

25. Zhang, S., Zhao, M., Bai, L., Hasegawa, T., Wang, J., Wang, L., Xue, H., Deng, Q., Xing, F., Bai, Y., Sakai, J., Bai, J., Koyanagi, R., Tsukumo, Y., Kataoka, T, Nagai, K., Hirose, K., and Ando, M., (2006). Bioactive guaianolides from siyekucai (Ixeris chinensis). J Nat Prod. 69: 1425-1428.

26. Zhou Y. X., Chen J., Li, J. P., Wang, Y. L., Jin, X. D. (2009). Chinese medicinal herbs in treating model rats with hepatic fibrosis. Afr J Tradit Complement

Altern Med. 7:104-108. 